\title{
SOME GEOMETRIC PROPERTIES OF MAGNETO-FLUID FLOWS
}

\author{
S.S. GANGWAR and RAM BABU \\ Department of Mathematics \\ Faculty of Science \\ Banaras Hindu University \\ Varanasi 221005, INDIA
}

(Received November 1, 1979)

\begin{abstract}
By employing an anholonomic description of the governing equations, certain geometric results are obtained for a class of non-dissipative magnetofluid flows. The stream lines are geodesics on a normal congruence of the surfaces which are the Maxwellian surfaces.

KEY WORDS AND PHRASES. Maxwellian Surfaces, Geodesics, Developal lis.
\end{abstract} 1980 MATHEMATICS SUBJECT CLASSIFICATION CODES: 76 W05.

1. INTRODUCTION.

In recent years in order to study the kinematic and kinetic properties of fluid flows, the attention of research investigators has been directed toward the application of differential geometry to fluid dynamics [1]-[9]. The characterization of a three-dimensional vector field in terms of anholonomic coordinates was first introduced by Vranceanu [10] and has been employed by Marris and Passman [5], [7] to describe some kinematic properties in hydrodynamics. G. Purushotham and his group [6], [9] have also used anholonomic geometric results in their study of fluid flows. 
This paper presents a kinematic study of the steady and non-dissipative magneto-fluid flows with the use of anholonomic geometric results. It has been shown that if at each point of the flow region the magnetic field line is in the plane of the stream line and its binormal, there exists a normal congruence of the surfaces which are the Maxwellian surfaces. The stream lines are the geodesics and their binormals are parallels on these surfaces. The speed and the fluid pressure are constant along the binormals of the stream lines. The Bernoulli equation in MHD has also been derived in a special case.

\section{THE BASIC EQUATIONS}

The basic equations governing the steady and non-dissipative magneto-fluid flows given by Lundquist [11] are as follows:

$$
\begin{aligned}
& \left(\rho v^{j}\right)_{j}=0, \\
& \rho v^{j} v_{i, j}+p,_{i}=\mu H^{j} H_{i, j}-\frac{1}{2} \mu\left(H^{2}\right),{ }_{i}, \\
& v^{j} \eta_{j}=0, \\
& \left(v^{j} H^{i}-H^{j} v^{i}\right)_{j}=0,
\end{aligned}
$$

and

$$
\mathrm{H}_{, j}^{\mathbf{j}}=0
$$

where $\mu$ is the constant magnetic permeability, $p$ the fluid pressure, $\rho$ the fluid density, $n$ the entropy of the fluid, $v^{j}$ the velocity field vector, and $H^{j}$ the magnetic field vector.

Using the substitution $h^{j}=\sqrt{\mathrm{H}^{j}}$ in (2.2), (2.4), and (2.5), we get respectively

$$
\begin{aligned}
& \rho v^{j} v_{i, j}+p^{*}{ }^{*}=h^{j} h_{i, j}, \\
& \left(v^{j} h^{i}-h^{j} v^{i}\right)_{, j}=0,
\end{aligned}
$$

and

$$
{ }^{h^{j}}, j=0,
$$


where $p^{*}=p+\frac{1}{2} h^{2}$ is the total pressure and $h^{2}=h^{j} h_{j}$. Equations (2.7) and (2.8) imply

$$
v^{j} h_{i, j}+h_{i} v_{j}^{j}-h^{j} v_{i, j}=0 .
$$

\section{ANHOLONOMIC GEOMETRIC RESULTS}

In this section we are going to state the results given by Marris and Passman [5]. If we consider the orthonormal basis $\left\{t^{i}, n^{i}, b^{i}\right\}$ introduced along the stream lines, where $t^{i}, n^{i}$, and $b^{i}$ are the unit vectors along the tangents, the principal normals, and the binormals of the stream lines, we have the results

$$
\begin{aligned}
& t^{j} t_{i, j}=k n_{i}, \\
& t^{j} n_{i, j}=-k t_{i}+\zeta b_{i},
\end{aligned}
$$

and

$$
t^{j} b_{i, j}=-\zeta n_{i}
$$

which are the Serret - Frenet formulae, where $k$ and $\zeta$ are the curvature and torsion of $t^{j}$-lines. The latter results are augmented by the intrinsic derivatives along $n^{j}-1$ ines and $b^{j}-1$ ines given by, in terms of the eight parameters $k, \zeta, \Omega_{t}, \Omega_{n}, n_{, j}^{j}, b_{j}^{j}, \theta_{n t}=n^{j} t_{, j}^{i} n_{i}, \theta_{b t}=b^{j} t_{, j}^{i} b_{i}$,

$$
\begin{aligned}
& n^{j} t_{, j}^{i}=\theta_{n t} n^{i}-\left(\zeta+\Omega_{n}-\Omega_{t}\right) b^{i}, \\
& n^{j} n_{, j}^{i}=-\theta_{n t} t^{i}-\left(b_{, j}^{j}\right) b^{i}, \\
& n^{j}{ }^{i}, j=\left(\zeta+\Omega_{n}-\Omega_{t}\right) t^{i}+\left(b_{, j}^{j}\right) n^{i}, \\
& b^{j} t_{, j}^{i}=-\left(\zeta+\Omega_{n}\right) n^{i}+\theta_{b t} b^{i}, \\
& b^{j} n_{, j}^{i}=\left(\zeta+\Omega_{n}\right) t^{i}+\left(k+n^{j}, j\right) b^{i},
\end{aligned}
$$

and

$$
b^{j} b_{, j}^{i}=-\theta_{b t} t^{i}-\left(k+n_{, j}^{j}\right) n^{i}
$$

where $\Omega_{t}$ and $\Omega_{n}$ are abnormalities of $t^{j}-1$ ines and $n^{j}-1$ ines respectively given by 


$$
\Omega_{t}=t_{i} e^{i j k}\left(t_{k}\right), j
$$

and

$$
\Omega_{n}=n_{i} e^{i j k}\left(n_{k}\right), j
$$

It should be noted that here we employ the parameter $\Omega_{n}$ instead of $\psi=\Omega_{b}-\Omega_{n}$ as employed by Marris and Passman; $\Omega_{b}$ the abnormality of $b^{j}$-lines, is expressed in terms of other parameters by the relation

$$
\Omega_{b}=-2 \zeta+\Omega_{t}-\Omega_{n}
$$

The further results are

$$
\begin{aligned}
& e^{i j k}\left(t_{k}\right)_{, j}=\Omega_{t} t^{i}+k b^{i}, \\
& e^{i j k}\left(n_{k}\right)_{, j}=-\left(b_{, j}^{j}\right)^{i}+\Omega_{n} n^{i}+\theta_{n t} b^{i}, \\
& e^{i j k}\left(b_{k}\right)_{, j}=\left(k+n_{, j}^{j}\right) t^{i}-\theta_{b t} n^{i}+\Omega_{b} b^{i},
\end{aligned}
$$

and

$$
t_{, j}^{j}=\theta_{n t}+\theta_{b t}
$$

\section{INTRINSIC PROPERTIES OF THE FLOW.}

Now we will derive some kinematic properties of magneto-fluid flows employing the results of earlier section. First we will decompose the basic equations governing the flow.

The velocity vector $v^{j}$ and magnetic field vector $h^{j}$ can be expressed as

$$
v^{j}=q t^{j},
$$

and

$$
h^{j}=h_{t} t^{j}+h_{n} n^{j}+h_{b} b^{j},
$$

where $q$ is the the magnitude of the velocity; and $h_{t}, h_{n}, h_{b}$ are the components of magnetic field vectors along $t^{j}, n^{j}, b^{j}$ respectively.

Using (4.1), (4.2) and the geometric results (3.1) - (3.9), (3.16) in the basic equations $(2.1),(2.6),(2.8)$ and $(2.9)$, we get the following decomposition

$$
t^{j}(\ln \rho q)_{, j}+\theta_{n t}+\theta_{b t}=0
$$




$$
\begin{aligned}
& \rho q t^{j} q_{, j}+t^{j} p_{, j}^{*}=-h_{t} h_{n} k+h_{n} h_{b}\left(2 \zeta+2 \Omega_{n}-\Omega_{t}\right) \\
& -h_{n}^{2} \theta_{n t}-h_{b}^{2} \theta_{b t}+h_{t} t^{j} h_{t, j} \\
& +h_{n} n^{j} h_{t, j}+h_{b} b^{j} h_{t, j} \text {, } \\
& \rho q^{2} k+n^{j} p_{, j}^{*}=h_{t}{ }^{2} k+h_{n} h_{t} \theta_{n t}-h_{b} h_{t}\left(\Omega_{n}+2 \zeta\right) \\
& +h_{n} h_{b} b_{, j}^{j}-h_{b}{ }^{2}\left(k+n_{, j}^{j}\right)+h_{t} t^{j} h_{n, j} \\
& +h_{n} n^{j} h_{n, j}+h_{b} b^{j} h_{n, j} \text {, } \\
& b^{j} p_{, j}^{*}=-h_{n} h_{t}\left(\Omega_{n}-\Omega_{t}\right)-h_{n}{ }^{2} b_{, j}^{j}+h_{b} h_{t} \theta_{b t} \\
& +h_{b} h_{n}\left(k+n_{, j}^{j}\right)+h_{t} t^{j} h_{b, j}+h_{n} n^{j} h_{b, j} \\
& +h_{b} b^{j} h_{b, j} \text {, } \\
& t^{j} h_{t, j}+n^{j} h_{n, j}+b^{j} h_{b, j}+h_{t}\left(\theta_{n t}+\theta_{b t}\right)+h_{n} n_{, j}^{j}+h_{b} b_{, j}^{j}=0, \\
& h_{n} n^{j} q_{, j}+h_{b} b^{j} q, j-q t^{j} h_{t, j}+q k h_{n}-q h_{t}\left(\theta_{n t}+\theta_{b t}\right)=0, \\
& h_{n} t^{j} q, j+q t^{j} h_{n, j}+q h_{b} \Omega_{n}+h_{n} q \theta_{b t}=0,
\end{aligned}
$$

and

$$
h_{b} t^{j} q, j+q t^{j} h_{b, j}+h_{b} q \theta_{n t}+h_{n} q\left(2 \zeta+\Omega_{n}-\Omega_{t}\right)=0 .
$$

Let us assume that the component of magnetic field vector in the direction of the principal normal of the stream line vanishes at each point of the flow region. Then the equations $(4.4 a)-(4.6 c)$ are reduced respectively to

$$
\begin{aligned}
& \rho q t^{j} q_{, j}+t^{j} p_{, j}^{*}=-h_{b}{ }^{2} \theta_{b t}+h_{t} t^{j} h_{t, j}+h_{b} b^{j} h_{t, j}, \\
& \rho q^{2} k+n^{j} p_{, j}^{*}=h_{t}{ }^{2} k-h_{b} h_{t}\left(\Omega_{n}+2 \zeta\right)-h_{b}{ }^{2}\left(k+n^{j}, j\right), \\
& b^{j} p_{, j}^{*}=h_{b} h_{t} \theta_{b t}+h_{t} t^{j} h_{b, j}+h_{b} b^{j} h_{b, j}, \\
& t^{j} h_{t, j}+b^{j} h_{b, j}+h_{t}\left(\theta_{n t}+\theta_{b t}\right)+h_{b} b_{, j}^{j}=0,
\end{aligned}
$$




$$
\begin{aligned}
& h_{b} b^{j} q_{, j}-q t^{j} h_{t, j}-q h_{t}\left(\theta_{n t}+\theta_{b t}\right)=0, \\
& q h_{b} \Omega_{n}=0,
\end{aligned}
$$

and

$$
h_{b} t^{j} q_{, j}+q t^{j} h_{b, j}+h_{b} q \theta_{n t}=0 \text {. }
$$

(4.9c) can be written as

$$
t^{j}\left(\ln q h_{b}\right)_{, j}+\theta_{n t}=0, \text { if } h_{b} \neq 0 .
$$

(4.8) and (4.9a) imply

$$
b^{j}\left(\ln q h_{b}\right)_{, j}+b_{, j}^{j}=0, \text { if } h_{b} \neq 0 .
$$

If $q=0$, the velocity vanishes; that means the fluid is stationary. [Since the fluid is not stationary, $q \neq 0]$. Therefore, (4.9b) implies

$$
\Omega_{\mathrm{n}}=0 \text {, if } \mathrm{h}_{\mathrm{b}} \neq 0 \text {; }
$$

that is, the abnormality of the principal normal vanishes which is the necessary and sufficient condition for the existence of normal congruence of the surfaces containing $t^{j}$-lines and $b^{j}$-lines. Since the vector $h^{j}$ is in the plane of $t^{j}$ and $b^{j}$, the magnetic field lines lie on these surfaces. Hence, the normal congruence of the surfaces is nothing but the family of the Maxwellian surfaces. Since the principal normals of the stream lines are the normals of these surfaces the stream lines are the geodesics. Thus we have the theorem:

THEOREM 1. If at each point of the flow region the component of the magnetic field vector in the direction of the principal normal of the stream line vanishes, there exists a normal congruence of the surfaces containing the stream lines and their binormals which are the geodesics and parallels respectively. These surfaces are the Maxwellian surfaces.

If the magnetic field lines coincide with the binormals of the stream lines and the density does not vary along the stream lines, the equations (4.3) (4.6c) will be put in the forms

$$
t^{j} q_{, j}+q\left(\theta_{n t}+\theta_{b t}\right)=0
$$




$$
\begin{aligned}
& t^{j}\left(\frac{1}{2} \rho q^{2}\right)_{, j}+t^{j} p_{, j}^{*}+h^{2} \theta_{b t}=0, \\
& \rho q^{2} k+n^{j} p_{, j}^{*}+h^{2}\left(k+n^{j}, j\right)=0, \\
& b^{j} p_{, j}=0, \\
& b^{j} \ln h, j+b^{j}, j=0, \\
& b^{j} q, j=0, \quad \text { if } h \neq 0, \\
& \Omega_{n}=0,
\end{aligned}
$$

and

$$
h t^{j} q_{, j}+q t^{j} h, j+h q \theta_{n t}=0 .
$$

Using (4.13) in $(4.16 c)$, we get

$$
t^{j} h_{, j}-h \theta_{b t}=0 \text {. }
$$

From (4.14c) and (4.16a) we have the theorem:

THEOREM 2. If the magnetic field lines coincide with the binormals of the stream lines, the fluid pressure and magnitude of the velocity are constant along the binormals.

Let us take the additional assumption that the magnetic intensity is constant along the stream lines, i.e. $t^{j} h_{, j}=0$, then (4.17) implies

$$
\theta_{b t}=0 \text {, }
$$

that is the geodesic curvature of $b^{j}-1$ ines vanishes. Thus we see that the magnetic field lines are the geodesics on the normal congruence of the surfaces, and hence we get the theorem:

THEOREM 3. If the fluid density and the magnetic intensity do not vary along the stream lines, the family of the Maxwellian surfaces is the normal congruence of developables. The stream lines and the magnetic field lines are orthogonal geodesics on these developables.

Employing (4.18) in (4.14a), we get 


$$
t^{j}\left(\frac{1}{2} \rho q^{2}\right), j+t^{j} p_{, j}^{*}=0
$$

that is

$$
\frac{\delta}{\delta t}\left(\frac{1}{2} q^{2}+p+\frac{1}{2} h^{2}\right)=0
$$

where $\frac{\delta}{\delta t}$ is the intrinsic derivative along the stream lines. Integrating (4.19), we get

$$
\frac{1}{2} \rho q^{2}+p+\frac{1}{2} h^{2}=\text { constant along the stream lines. }
$$

This is the Bernoulli equation.

\section{REFERENCES}

[1] Truesde11, C. Intrinsic Equations for Spatial Gas Flows, Zeits. Angew. Math. Mech., 40 (1960), 9-14.

[2] Kanwal, R.P. Intrinsic Relations in Magnetohydrodynamics, Zeits. Angew. Math. Mech., 41 (1961), 462-463.

[3] Suryanarayan, E.R. On the Geometry of Stream Lines in Hydromagnetic Fluid Flows When the Magnetic Field is Along a Fixed Direction, Proc. Amer. Math. Soc., 16 (1965), 90-96.

[4] Wasserman, R.H. On the Geometry of Magnetohydrodynamic Flows, Quart. J. Mech. App1. Math., 20 (1967), 219-231.

[5] Marris, A.W. and Passman, S.L. Vector Fields and Flows on Developable Surfaces, Arch. Rational Mech. Anal. 32 (1969), 29-86.

[6] Purushotham, G. and Rao, Gopal Krishna On the Geometry of Vertex Line in Viscous Incompressible Steady Flows, Tensor, 23 (1972), 131-138.

[7] Marris, A.W. Steady Universal Motion of a Navier-Stokes Fluid: The Case When the Velocity Magnitude is Constant on a Lamb Surface, Arch. Rational Mech. Anal., 50 (1973), 99-110.

[ 8] Indrasen, Steady Rotational Hydromagnetic Flows, Tensor, 32 (1978), 350354.

[9] Purushotham, G. and Aswarthanarayana, On the Vertex - Line Geometry in Pseudo-Stationary Spatial Gas Flows, Tensor, 33 (1979), 11-20.

[10] Vranceanu, M.G. "Les Espaces non Holonomes," Memorial des Sciences Mathematiques, Paris, 76 (1936).

[11] Lundquist, S. Studies in Magnetohydrodynamics, Ark Fys., 5 (1952), 297. 


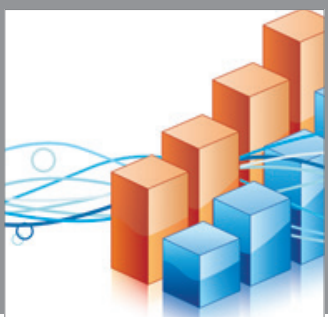

Advances in

Operations Research

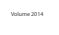

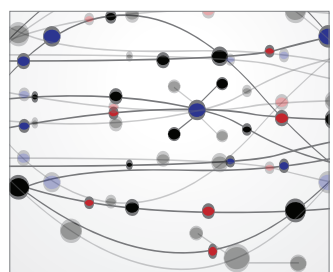

\section{The Scientific} World Journal
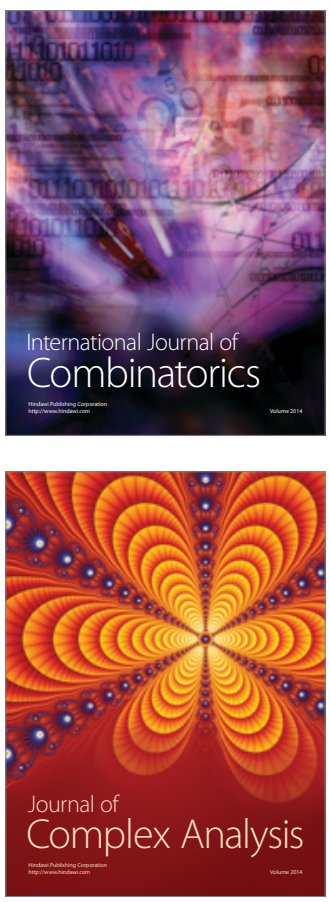

International Journal of

Mathematics and

Mathematical

Sciences
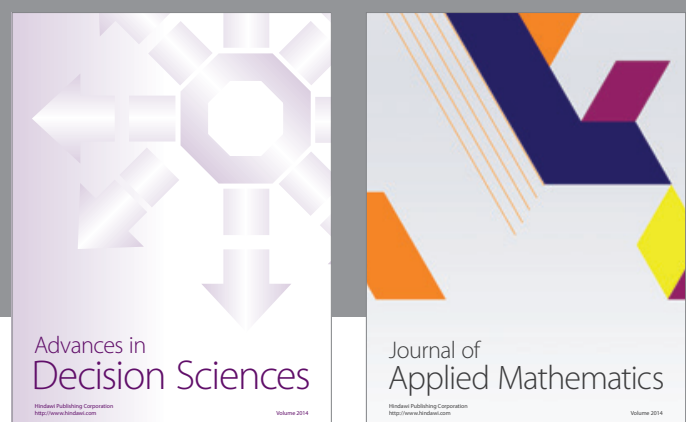

Journal of

Applied Mathematics
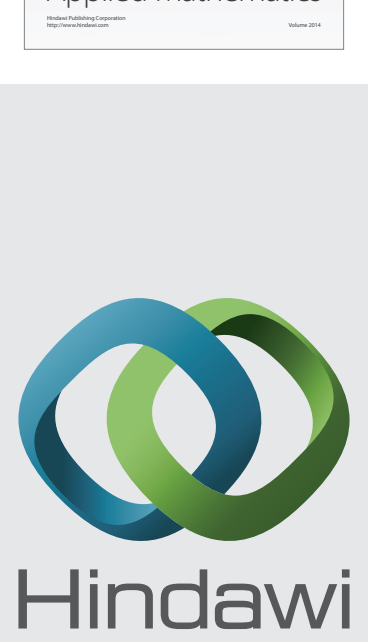

Submit your manuscripts at http://www.hindawi.com
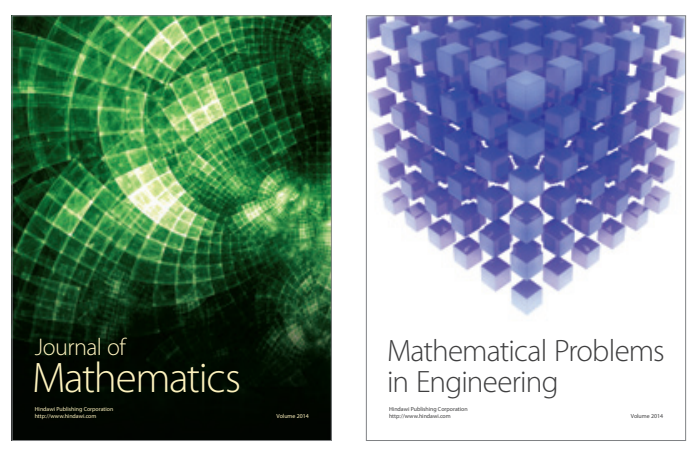

Mathematical Problems in Engineering
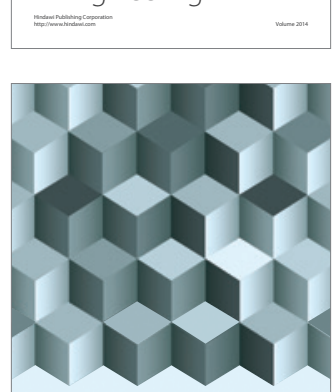

Journal of

Function Spaces
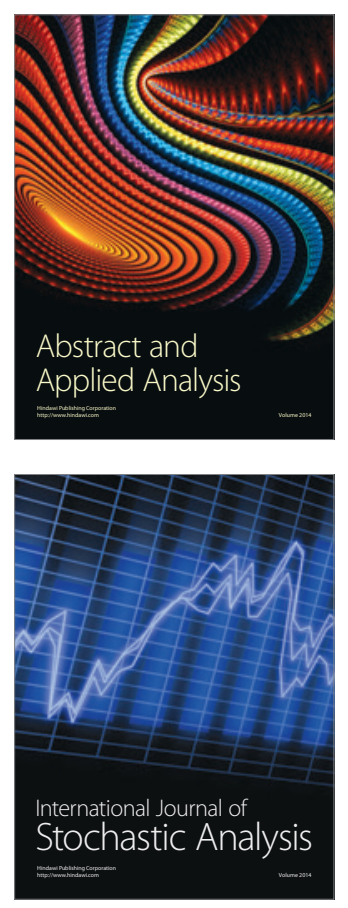

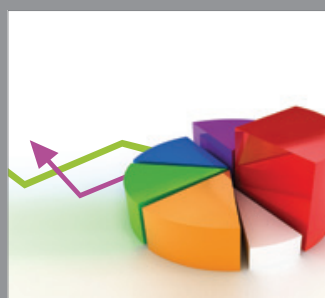

ournal of

Probability and Statistics

Promensencen
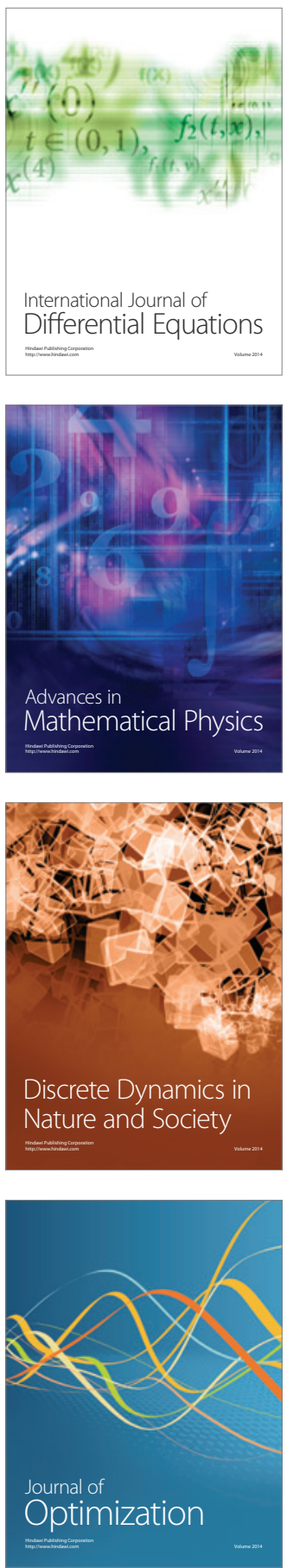